\title{
Über das Alter der georgischen Übersetzung des Neuen Testamentes.
}

\section{Von Theodor Kluge.}

Ein Gebiet der neutestamentlichen Theologie, das leider außerordentlich wenig begangen ist, ist das der georgischen Übersetzungen neutestamentlicher Schriften; aber nicht bloß allein diese kommen in Betracht, sondern auch die alttestamentlicher, und dann nicht zu vergessen die Schriften der Kirchenväter, Heiligenlegenden und anderes, was damit zusammenhängt. Daß das Ganze von vornherein wenig Interesse beanspruchen dürfte, kann man nicht mehr sagen, nachdem Bonwetsch einiges veröffentlicht hat (leider nur nach russischen Übersetzungen), das um so größeres Interesse beansprucht, weil es eben nur durch das Georgische uns erhalten ist. $\mathrm{Zu}$ verwundern ist das freilich nicht, denn es gehört zưr Beurteilung solcher Dinge einmal eine große Menge theologischer Kenntnisse, die dem Sprachforscher nicht geläufig sind, und der Theologe muß zwei neue Sprachen oder drei - armenisch, georgisch, syrisch - lernen, wenn seine Arbeiten nicht einfach wertlos werden sollen.

Für mich als Nichttheologen kommt das erstere in Betracht, und der Grund, weshalb ich hier meine Kleinigkeit vorbringe, liegt darin, daß ich nicht einzusehen vermag, warum die eine Wissenschaft der anderen nicht bei Gelegenheit beispringen soll. Der Weg, auf dem ich überhaupt zu der Frage gelangt bin, ist der: Vor einiger Zeit bearbeitete ich für ein historisches Lexikon des Georgischen als Vorarbeit die indogermanischen Lehnwörter nach dem Dictionnaire géorgien-russefrançais de D. Tšubinov. Dabei stellte sich als unabweisbare Notwendigkeit heraus; diese Lehnwörter nach den Quellenschriften zu bearbeiten, und so fing ich mit den vier Evangelien, dem ältesten georgischen Text, an. Bevor ich die Resultate meiner Arbeit mitteile, will ich kurz die Arbeiten zweier Vorgänger besprechen ${ }^{1}$. Es sind

1 Auf die Arbeiten der Georgier selbst und auch die russischer Gelehrter kann ich nicht eingehen; ein Teil von ihren Arbeiten ist mir nicht zugänglich, sie zu beschaffen geht nicht mehr, denn es fchlt mir dazu an Zeit. 
das dic Arbciten von P'almieri im Bessarione ser. II, 6 (1904) und von H. Goussen im Oriens Christianus $\sigma$ (1906). Beide haben als Titel: dic georgische Bibclübersetzung. An sich schon muß dieser 'Titel zu Irrtümern Veranlassung geben. Der Artikel Palmieris beruht offenbar auf einem älteren Aufsatz oder Aufsätzen Chachanovs und enthält nichts, was dic Lösung der Frage bringt. Dem gegenüber bildet die Arbeit Goussens einen wesentlichen Fortschritt. Indem sie erstens den landläufigen: $d$. h. unter den Georgiern verbreiteten Irrtum als solchen nachweist, nämlich, daß die georgische Übersetzung vor der armenischen und unabhängig davon entstanden ist, und zweitens, daß auch schon das Neue Testament, ganz abgesehen von einer Bibelübersetzung, stückweise und nach und nach übersetzt worden ist. Der sprachivissenschaftliche Nachweis dafür weiter unten.

Die Frage ist nur wann. Es ist die Tendenz bei allen georgischen " "Historikern“ und Literarhistorikern, die Übersetzung nur möglichst hoch hinaufzuschrauben und sie älter sein zu lassen als die armenische.

Ist das letztere nun schon wenig wahrscheinlich, so fällt die erste Behauptung aus dem Grunde fort: Wir haben eine Reihe von georgischen Inschrifter an Kirchen, die Brosset herausgegeben hat und deren älteste aus dem Jahre 953 (die Zahl ist mir nur in der Erinnerung) stammt. Wie ist nun diese enorme Differenz von fast einem halben Jahrtausend $z \mathrm{u}$ erklären? Einfach dadurch, daß die georgische Übersetzung um fast ebensoviel herunterzurücken ist, und das folgt aus einer Betrachtung zunächst der Eigennamen und dann der griechischen und armenischen Lehnwörter, die in der Übersetzung enthalten sind.

Erschwert wird die Untersuchung nur einmal dadurch, daß es keine kritische Ausgabe des georgischen neuen Testamentes gibt und dann, daß die armenische ebenfalls nur mehr einen Ersatz findet in der Sohrapschen Ausgabe. Beide Forderungen sind zwar vorläufig entbehrlich, da die vorhandenen Hilfsmittel ausreichen, um die Frage mit hinlänglicher Sicherheit zu entscheiden. Für meine Untersuchung habe ich benutzt eine Ausgabe der br. Bibelgesellschaft: 4 Evangelien, Tiflis 1900; ein Neues Testament derselben Gesellschaft, Tiflis 1879. Wie ich durch Stichproben festgestellt habe, geht diese auf die in meinem Besitz befindliche große Moskauer Ausgabe von 1816 zurück. Für das Armenische habe ich die Ausgabe altarm. Constantinopel 1896 benutzt.

. Von vornherein will ich bemerken, daß neben der griechischen und armenischen Ausgabe auch aller Wahrscheinlichkeit nach noch sekundär eine syrische Übersetzung herangezogen worden ist; sowie ich Zeit habe, will ich auch das genauer nachprüfen. Ebenso ist mir vollkommen 
klar, daß die georgische Übersetzung - aller Wahrscheinlichkeit nach später (oder früher?) - noch einmal aus dem Griechischen übersetzt worden ist; es geht das aus dem Lehnwörterauszuge aus dem Tš'schen Lexikon hervor, in dem sich griechische Lehnwörter finden, die nur im Neuen Testament vorkommen können, und die ich bis jetzt nicht darin habe belegen können, es sei denn mit georgischen Wörtern. Ich habe mir die Mühe gemacht, nur eine einwandfreie Untersuchung der Lehnwörter zu liefern, und habe sämtliche Eigennamen des Neuen Testamentes in einer Tabelle armenisch-georgisch-griechisch zusammengestellt (die Arbeit erscheint im laufenden Jahrgang der Ztschr. für vergl. Sprachwissenschaft). So interessant allein sprachgeschichtlich das Studium dieser vergleichenden Tabellen ist, so will ich hier nur einige Hauptpunkte mitteilen.

Es ist klar: wenn man von vornherein mit so unzureichenden Mitteln arbeiten muß, so kann nicht viel dabei herauskommen. Das Markus-Evangelium schließt mit c. 16, 20. Joh 7,53 bis 8, 11 steht ebenfalls an der gewöhnlichen Stelle. Dagegen fehlt Hebr. 11,24. Das sind die drei Hauptunterschiede gegen die Opiser-Handschrift des AthosKlosters (cfr. 'Goussen, 1. c.).

Von anderen Abweichungen finden sich in meinen Texten noch folgende:

Mt 3, 7 steht parisewelni da sadukewelni in der arm. Version in umgekehrter Folge.

Mit 14, 2, qrmebisa da dedebisa, umgekehrt gr.: ruvaıkŵv kai maıðíwv. Mit 16, 2-4 fehlt im Arm.; gr.: ówíac-dúvac $\theta \epsilon$.

Mt 20, 2 ebenfalls Abweichung.

Mt 23,14 desgl.

Mit 23, 25 Zusatz nur im Georgischen + da pinakisasa.

Mt 27, 35+iva $\pi \lambda \eta \rho w \theta \hat{n} \kappa \tau \lambda$. im Text.

Markus: Abweichungen gegen den Annischen Text:

Nk 1,34; 3, 8; 7,8; 13,2.8.

gegen den gr. Text 7,$8 ; 9,44.46 .48 ; 10,21$.

Lukas: Abw. gegen den arm. Text.

Lk 1, 13; 10, 41 .

gegen den gr. Text:

Lk 16, 23; 24, 12.

Johannes -.

Das ist im wesentlichen das, was mir während meiner Arbeit auffiel. Es kann sich ja in diesen Fällen immer nur um zwei Sachen handeln; entweder um Auslassungen oder Zusätze, da ja durch die 
Übersetzung die feineren Unterschiede im griechischen Text selbst ohne weiteres verloren gehen. $\mathrm{Da}$ sich mein Hauptaugenmerk nicht darauf richtete, so gebe ich von vornherein $z u$, daß ich Stellen übersehen habe.

Von Interesse ist, daß sich aus den wenigen Stellen schon feststellen läßt, welche griechischen Handschriften wenigstens mittelbar in Frage kommen. Aus Mk 9, 44. 46. A und D (v. Soden $\delta 4,5)$ Lk 24, 12 $T$ (v. Soden $\in G$ ).

Einen viel intimeren Einblick in das Getriebe dieser Ubersetzung gewährt nun die Untersuchung der Eigennamen. $\mathrm{Da}$ ist nun zunächst ein ganz fundamentaler Unterschied zwischen der Wiedergabe der Eigennamen aus der griechischen Vorlage und der aus der armenischen. Eine ganz geringe Anzahl von Wörtern ist selbständig, ich zähle nachher einige auf. Dieser Unterschied besteht darin, daß die Wiedergabe aus der griechischen Vorlage schwankt, aus der armenischen nicht. Die letztere scheidet daher aus dem Kreise unserer Betrachtungen aus. Es ergibt sich weiterhin, da $\beta$ die Kenntnis der armenischen Übersetzer in betreff des Griechischen bedeutender war als die der georgischen. Da ist zunächst das Schwanken in der Wiedergabe des gr. $\beta$ : B $\alpha \lambda \alpha ́$ - -walak;

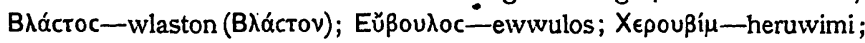
dann die Wiedergabe von $r$ als $g$ gewöhnlich, als $\breve{g}=$ hebr. $y$ in

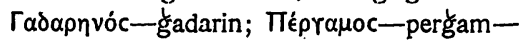

ferner die von $\epsilon$ auffallenderweise:

$\epsilon=\mathrm{i}$.

'Av১péac Mt u. Lk andria; 'Apeorareítnc-ario- Act.

Was indessen durchschlagend gegen die vorarmenische Übersetzung spricht, ist die Wiedergabe des gr. $\eta$ durch georg. $i$, während armenisch $\eta=e$ ist, und zwar ausnahmelos.

Ferner ist ein Schwanken zu konstatieren bei der Wiedergabe von $. c=s$ und $z ; o=0$ und $u ; v=w i, u, i, \alpha=e$ und $i, o v=w i, o, u$, $\hat{\mathrm{u}} ; \epsilon \mathfrak{l}=\mathrm{i}, \epsilon \mathrm{U}=\mathrm{ew}) ; \chi=\mathrm{k}^{\mathrm{k}}$ und $\mathrm{h}$ in der armenischen Übersetzung ist $\mathrm{x}$ durch $\mathrm{k}(=\mathrm{k}+\mathrm{h})$ wiedergegeben mit einer einzigen Ausnahme, trotzdem es im Armenischen einen dem gr. $\chi$ entsprechenden Laut gibt. Xap̉ódv arm. xaran $(\mathrm{x}=\mathrm{x})$.

Die Frage ist nur die: woher kommt das? Diese läßt sich in zweifacher Hinsicht erklären, einmal durch die Differenz zwvischen dem geschriebenen Griechisch, und dem, welches im Kaukasus oder von den Leuten, die dorthin gekommen waren, gesprochen wurde, sei es nun, $\mathrm{da} B$ diese Leute von Byzanz, oder über Armenien kamen, oder auch, was jedoch wenig wahrscheinlich, griechisch sprechende Armenier oder aber von eingeborenen Griechen, aus Kolonien am schwarzen Meer aus 
altgriechischer Zeit waren. Das reicht aber nicht aus. Seinen zweiten Grund muß das Schwanken haben darin, daß uns die Schriften des NT. aus verschiedenen Zeiten vorliegen, und zwar sind schon die Evangelien nicht homogen, deutlich treten die Differenzen schon in der Apostelgeschichte hervor und werden gegen den Schluß des NT. hin immer bedeutender.

Von Einzelheiten auf dem Gebiet der Eigennamen führe ich noch folgende an, da sie einiges Interesse bieten und hier und da Aufschluß erteilen über die in Betracht kommenden Handschriften.

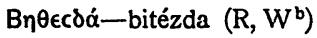

$\Sigma \mu u ́ p v \alpha-z m i r i n i s a(T$.)

und einige andere. Auffallenderweise Codd. des 5. und 6. Jahrh., $\mathrm{W}^{\mathrm{b}}$ (cfr. Nestle in der Einl.) 8-13. Interessant ist, daß der Name Kupia 2 Joh 1,5 in der armenischen Übersetzung als Eigenname aufgefaßt wird, in der georgischen dagegen nicht mehr: kiwria-dedopalsa (= Königin, Herrin).

An einigen Stellen ist die georgische Übersetzung völlig - selbständig, so Act 8, 27 Aibiow = indo d. h. = den asiatischen Aethiopen ${ }^{2}$ (cfr. Herodot III, 94. VII, 70) auch der Ausdruck für Höhle ist neu, und manches andere mehr.

Von den ca. 600 Eigennamen des NT. sind 200 aus der griechischen, der Rest aus der armenischen Vorlage entrommen.

Im einzelnen geben die Eigennamen recht unangenehme Rätsel auf, die sich leider vermehren, sobald man an die eigentliche Lehnwörterfrage herangeht. Diese habe ich leider erst für die vier Evangelien untersuchen können, da mir die Arbeit auf die Nerven fiel.

Einen weiteren Übelstand, den alle Lehnwörter an sich haben, ist die Frage: wann sind sie entlehnt? Von einem Wort wie 'Pódn ist schwerlich anzunehmen, daß es den Georgiern eher bekannt war, als

- sie die Übersetzung anfertigten; das kann man von anderen Wörtern nicht behaupten. Von den uns hier angehenden Wörtern muß nun aber leider behauptet werden, daß sie z.T. schon lange vor der Übersetzung existiert haben, und zwar - man sieht es ihnen an - sind sie wie zwar jedes Lehnwort zuerșt Fremdwort gewesen, aber auch geblieben, z. B. ká $\mu \eta \lambda$ oc georg. aklemi usw. Ich habe für die Evangelien im ganzen 35 griechische Lehnwörter feststellen können, und befremdend an ihnen ist, $\mathrm{da} ß$ in allen von ihnen das $\eta$ als e erhälten ist; die Wörter aber

1 [Eine junge Tradition nennt den Kammerherrn Indich (vgl. Laurent, Neutest. Studien $145 \mathrm{f}$.). $\mathrm{Da}$ muB irgendein Zusammenhang obwalten. E. P.] 
auch durch das Armenische nicht hindurchgegangen sind. Vor allem ist beachtenswert ekklesia "Gemeine" mit noch erhaltenem doppel-k (Doppelkonsonanzen im Griechischen werden regelmäßig aufgelöst), dagegen paraskcivi die einzige Ausnahme und bedeutend jünger.

Also zu einem unantastbaren Resultat ist nicht zu gelangen. Vielleicht wenn man mehrere Handschriften miteinander vergleichen könnte und vor allen Dingen palaeographische Methoden da wären, dann ließe sich viclleicht mehr damit anfangen. Hoffentlich bietet der Rest des NT. oder das AT., von dem wir wissen, daß es jünger ist, weitere Mittel zur Datierung, und das was mit diesem Wenigen erreicht ist, ist schließlich auch nicht zu verachten.

Die obere und untere Grenze liefern nur die armenischen Lehnwörter, auch hier tritt wieder ein bedenklicher Fall auf. Das Wort für „essen" wird im Georg. stets wiedergegeben durch žam, im Arm. aber durch owtem. Bei der Häufigkeit des Wortes ist das um so auffallender. žam kommt nun zwar schon, wie mir Herr Prof. Finck mitteilt, im Altarmenischen vor im "Leben der Väter", dessen älteste Teile zwar in das 4. Jahrhundert fallen, das aber erst im 12. Jahrhundert abgeschlossen wurde. Festzustellen, wann die einzelnen Teile entstanden sind, ist eine Aufgabe für sich, ich habe kein Interesse daran, umsomehr als es hier wie überall auf diesem Gebiet auf eine Menge Arbeit und minimale Resultate herauskommen wird. Der Rest der Wörter bietet keinen weiteren Anhalt.

Das ist im wesentlichen das, was ich mitzuteilen habe, und von dem ich glaube, daß es für Theologen von Interesse sein wird; $z u$ wünschen würde allerdings sein, daß etwas mehr als bisher auf diesem entlegenen Gebiete gearbeitet würde.. Aber ehe das ganze Gebiet nicht von Historikern in Angriff genommen wird, damit man überhaupt erst .einmal auf die Quellen kommt, und in den ganzen Wust von der hl. Nina und allen den anderen wie Noa, Mesech u. v. a. Einsicht bekommt, wird wenig $z \mathrm{u}$ erhoffen sein. Beiseite schieben kann man die Erledigung derartiger Fragen nicht und sie als historische Tatsachen hinnehmen geht erst recht nicht. Ich werde im Laufe des Jahres die Arbeit fortsetzen und, falls sich etwas Wertvolles herausstellen solite, das gelegentlich mitteilen.

Vorläufig ergibt sich nur das eine sichere Resultat: die georgische Übersetzung des NT., d. h. die Ausgabe, die ich untersucht habe, ist jünger als die armenische, um wieviel ist noch die Frage. 